\title{
Disorder-induced electronic nematicity
}

Steffensen, Daniel; Kotetes, Panagiotis; Paul, Indranil; Andersen, Brian

Published in:

Physical Review B

DOI:

10.1103/PhysRevB.100.064521

Publication date:

2019

Document version

Publisher's PDF, also known as Version of record

Citation for published version (APA):

Steffensen, D., Kotetes, P., Paul, I., \& Andersen, B. (2019). Disorder-induced electronic nematicity. Physical Review B, 100(6), [064521]. https://doi.org/10.1103/PhysRevB.100.064521 


\title{
Disorder-induced electronic nematicity
}

\author{
Daniel Steffensen, ${ }^{1}$ Panagiotis Kotetes,,${ }^{1,2}$ Indranil Paul, ${ }^{3}$ and Brian M. Andersen ${ }^{1}$ \\ ${ }^{1}$ Niels Bohr Institute, University of Copenhagen, Vibenshuset, Lyngbyvej 2, DK-2100 Copenhagen, Denmark \\ ${ }^{2}$ CAS Key Laboratory of Theoretical Physics, Institute of Theoretical Physics, Chinese Academy of Sciences, Beijing 100190, China \\ ${ }^{3}$ Laboratoire Matériaux et Phénomènes Quantiques, Université de Paris, CNRS, F-75013, Paris, France
}

(Received 11 June 2019; published 22 August 2019)

\begin{abstract}
We expose the theoretical mechanisms underlying disorder-induced nematicity in systems exhibiting strong fluctuations or ordering in the nematic channel. Our analysis consists of a symmetry-based Ginzburg-Landau approach and associated microscopic calculations. We show that a single featureless pointlike impurity induces nematicity locally, already above the critical nematic transition temperature. The persistence of fourfold rotational symmetry constrains the resulting disorder-induced nematicity to be inhomogeneous and spatially average to zero. Going beyond the single impurity case, we discuss the effects of finite disorder concentrations on the appearance of nematicity. We identify the conditions that allow disorder to enhance the nematic transition temperature, and we provide a concrete example. The presented theoretical results can explain a large series of recent experimental discoveries of disorder-induced nematic order in iron-based superconductors.
\end{abstract}

DOI: 10.1103/PhysRevB.100.064521

\section{INTRODUCTION}

The study of electronic nematic quantum phases [1] is becoming increasingly important in condensed matter systems due to a growing class of recently discovered materials exhibiting nematic behavior, i.e., spontaneous generation of spatial anisotropy. Nematicity has been experimentally identified in a number of correlated quantum materials [1], including quantum Hall states in higher Landau levels of two-dimensional (2D) electron gases [2,3], bilayer strontium ruthenates [4], cuprate high-temperature superconductors (SCs) [5], doped $\mathrm{Bi}_{2} \mathrm{Se}_{3}$ SCs [6-8], Fe-based SCs (FeSCs) [9-22] and, possibly, twisted bilayer graphene [23]. Thus, nematicity begins to establish as a universal electronic state of matter, which motivates further theoretical studies of its distinct properties.

Nematic phases are particularly prevalent in FeSCs, where experimental evidence for electronic nematicity comes from a wide range of techniques, including transport measurements [9-15], angle-resolved photoemission spectroscopy [16], scanning tunneling spectroscopy [17], neutron scattering [18], light spectroscopy [19,20,24], Andreev-point-contact measurements [21], and torque magnetometry [22]. In this case, the emergence of nematicity refers to the spontaneous breaking of fourfold $\left(C_{4}\right)$ rotational symmetry. Notably, the identification of the driving mechanism of nematicity in these systems is complicated, due to the coupling of spin, orbital, and lattice degrees of freedom at temperatures $(T)$ below the tetragonal-to-orthorhombic structural phase transition occurring at $T=T_{s}$ [25]. Particularly, the origin of nematicity in FeSe remains controversial at present [26].

The growing ubiquity of nematic correlated electronic systems that are scarcely free from impurities, calls for resolving the influence of disorder on the emergence of nematicity. In fact, the strong relevance of disorder to the nematic ordering is also supported from a notable number of experiments detecting local $C_{4}$ symmetry breaking around impurities [17,27-32]. While some of these results may be attributable to, for instance, residual sample strain which explicitly breaks the $C_{4}$ symmetry locally [32-34], or the presence of stripeordered antiferromagnetism [27], the possible pinning of nematic fluctuations due to the presence of disorder appears as a promising and, at the moment, poorly explored alternative [35-39]. Disorder has also been proposed to play an important role in generating split structural (nematic) and magnetic transitions in some FeSCs [40]. Even more, there are strong indications for disorder-pinned static local nematicity in the bulk tetragonal phase, i.e., above $T_{s}$ [22,41-46]. For example, two recent NMR experiments on FeSe [43,44] found a clear splitting and broadening of the NMR line shape above $T_{s}$. The presence of short-range nematic order above the bulk $T_{s}$ in FeSe has also been inferred from ARPES and opticalpump conductivity measurements [47,48]. Finally, two very recent pair distribution function (PDF) measurements of $\mathrm{FeSe}$ found clear evidence of pronounced local orthorhombicity at the length scale of a few nanometers well above $T_{s}[49,50]$, thus providing additional evidence for disorder-induced local nematicity in these systems.

Here we perform a detailed theoretical study of the role of disorder in systems with $D_{4 h}$ point-group symmetry, which additionally feature strong fluctuations or ordering in the nematic channel. The emergence of nematicity is reflected in a nonzero field $N$, which transforms according to the $B_{1 g}$ irreducible representation (IR) of $D_{4 h}$. We mainly focus on $T$ above the respective $T_{\text {nem }}$ (same as $T_{s}$ ), at which the spontaneous thermodynamic $C_{4}$ symmetry breaking takes place. By employing both phenomenological and microscopic approaches, we address the following three questions: (1) Under what circumstances can disorder generate nematicity locally? (2) What is the spatial profile of the resulting nematic-defect structure? (3) How do finite disorder concentrations influence the nematic transition? 
Our main results can be summarized as follows. For $T>$ $T_{\text {nem }}$, (i) an impurity potential of arbitrary strength with a spatial profile which respects the $C_{4}$ symmetry, generates a local nematic field $N(\boldsymbol{r})$ with a spatial profile belonging to the $B_{1 g}$ IR. By transferring to a polar coordinate system $(x, y) \mapsto$ $(r, \phi)$, this yields the spatial profile $N(r, \phi) \propto \cos (2 \phi)$. (ii) This further implies that a potential with a $C_{4}$-symmetric profile does not induce net nematicity, i.e., $\int d \boldsymbol{r} N(\boldsymbol{r})=0$, but local probes may still detect evidence of $C_{4}$-symmetry breaking. (iii) However, we show that such a potential can still drive a nematic transition already at $T>T_{\text {nem }}$, since it modifies the Stoner criterion for the nematic instability. (iv) A $C_{4}$-symmetry-breaking impurity potential can induce nonzero net nematicity and, thus, stabilize long-range nematic order. For $T<T_{\text {nem }}$, an impurity potential with a spatial profile which respects $C_{4}$ symmetry modifies the bulk nematicity $\left(N_{\mathrm{B}}\right)$ locally, and results in an inhomogeneous nematic field $N(\boldsymbol{r})=N_{\mathrm{B}}+\delta N(\boldsymbol{r})$, with a $\delta N(r, \phi)$ which is generally not proportional to $\cos (2 \phi)$.

\section{PHENOMENOLOGICAL GINZBURG-LANDAU APPROACH}

We first examine the implications of disorder within a continuum Ginzburg-Landau (GL) approach that allows exposing generic features of the induced nematic field, i.e., independent of the origin of the electronic nematicity. In fact, our GL results also apply to situations where the nematic field originates from the spontaneous mixing of superconducting order parameters belonging to the $A_{1 g}$ and $B_{1 g}$ IRs [51,52]. However, there, one has to further include the possible influence of disorder on the pairing.

The free energy density $\mathcal{F}(\boldsymbol{r})$ is a functional of $N(\boldsymbol{r})$ and the disorder potential $V(\boldsymbol{r})$. Its invariance under $D_{4 h}$ point group operations and time reversal, leads to

$$
\begin{aligned}
\mathcal{F}(\boldsymbol{r})= & \alpha\left(T-T_{\text {nem }}\right)[N(\boldsymbol{r})]^{2} / 2+\beta[N(\boldsymbol{r})]^{4} / 4 \\
& +c[\nabla N(\boldsymbol{r})]^{2} / 2+g N(\boldsymbol{r})\left(\partial_{x}^{2}-\partial_{y}^{2}\right) V(\boldsymbol{r}),
\end{aligned}
$$

with $\alpha, \beta, c>0$. Here we restricted to the lowest-order possible coupling between $V(\boldsymbol{r})$ and $N(\boldsymbol{r})$. Later on, we consider effects of higher-order terms. For further details on the GL approach, we refer to the Supplemental Material (SM) [53]. From Eq. (1), one observes that the nematic field couples to the second derivatives of the disorder potential and, thus, to a particular linear combination of the electric field gradients (EFGs). The nematic field is proportional to the quadrupolar electronic charge density defined as $Q_{x^{2}-y^{2}}(\boldsymbol{r})=\left(x^{2}-\right.$ $\left.y^{2}\right) \rho(\boldsymbol{r})$, which transforms according to the $B_{1 g}$ IR of $D_{4 h}$, i.e., similar to $N(\boldsymbol{r})$. In the above, $\rho(\boldsymbol{r})$ denotes the electric charge density, which belongs to the trivial $\left(A_{1 g}\right)$ IR of $D_{4 h}$. The appearance of a nonzero $N(\boldsymbol{r})$, solely due to the presence of disorder, is a consequence of the broken translational invariance, and can be viewed as a result of linear response, since the EFG $\left(\partial_{x}^{2}-\partial_{y}^{2}\right) V(\boldsymbol{r})$ acts as a quadrupolar source field, which leads to a nonzero and necessarily inhomogeneous $Q_{x^{2}-y^{2}}(\boldsymbol{r})$ and thus $N(\boldsymbol{r})$.

For the remainder, we consider $T>T_{\text {nem }}$ (unless explicitly stated), which implies that the system resides in the $C_{4}$-symmetric phase in the absence of disorder. In this case,
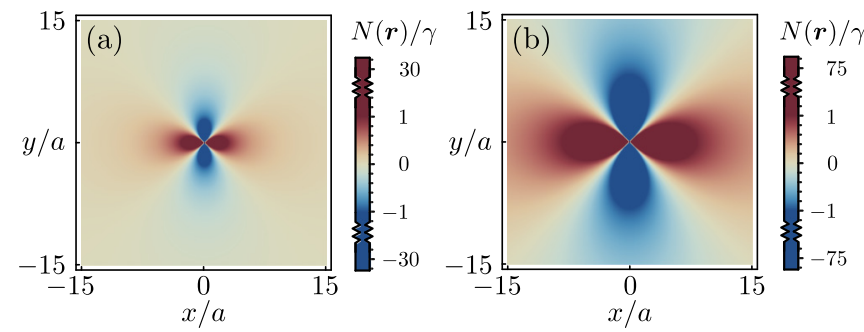

FIG. 1. (a) Nematic order parameter $N(\boldsymbol{r})$ at $T \gg T_{\text {nem }}$, where $\xi_{\text {nem }} \sim 5 a$. (b) Same as in (a), but with $T \gtrsim T_{\text {nem }}$ resulting in a larger nematic coherence length $\xi_{\text {nem }} \sim 15 a$. The figures were obtained using Eq. (3) with a convenient impurity profile of the form $V(\boldsymbol{r})=$ $V /|\boldsymbol{r}|$, without loss of generality. We introduced $\gamma=-\pi g V /\left(2 \xi_{\text {nem }}\right)$ and used $c=1$.

we can drop the quartic nematic term, since $N(\boldsymbol{r})$ is generally small. Thus, for $T>T_{\text {nem }}$, the Euler-Lagrange equation of motion (EOM) for Eq. (1) reads

$$
\left[\alpha\left(T-T_{\text {nem }}\right)-c \nabla^{2}\right] N(\boldsymbol{r})=-g\left(\partial_{x}^{2}-\partial_{y}^{2}\right) V(\boldsymbol{r}) .
$$

The above EOM provides the proportionality relation between the EFG and the resulting nematic field, i.e.,

$$
N(\boldsymbol{r})=\int \frac{d \boldsymbol{q}}{(2 \pi)^{2}} e^{i q \cdot r} \frac{g}{c} \frac{\left(q_{x}^{2}-q_{y}^{2}\right) V(\boldsymbol{q})}{\boldsymbol{q}^{2}+\xi_{\mathrm{nem}}^{-2}},
$$

where we introduced the nematic coherence length in the tetragonal phase $\xi_{\text {nem }}^{-1}=\sqrt{\alpha\left(T-T_{\text {nem }}\right) / c}$.

For a $C_{4}$-symmetric impurity potential we integrate the angular part of the right-hand side (rhs) in Eq. (3), and find the earlier-announced spatial profile $N(r, \phi) \propto \cos (2 \phi)$. This profile decays away from the impurity within a range given by $\xi_{\text {nem }}$, and this length scale diverges as $T \rightarrow T_{\text {nem. }}$. Both results are depicted in Fig. 1. The angular dependence transforms exactly according to the $B_{1 g}$ IR of $D_{4 h}$. This constraint on the spatial profile of $N(\boldsymbol{r})$ is a direct consequence of the featureless $\left(A_{1 g}\right)$ nature of the disorder potential $V$ itself. Thus, the net electronic nematicity and quadrupolar charge are zero, since

$$
\int d \boldsymbol{r} N(\boldsymbol{r})=N(\boldsymbol{q}=\mathbf{0}) \propto \int_{0}^{2 \pi} d \phi N(r, \phi)=0 .
$$

Nonetheless, probes like NMR and PDF pick up a signal from atoms in the lobes of the induced $N(\boldsymbol{r})$, and do therefore detect clear evidence for local nematicity and orthorhombicity even though global effects are absent.

Equation (4) also reveals that the linear coupling of the nematic field to the EFG cannot stabilize a net thermodynamic nematicity which emerges when $N(\boldsymbol{q}=\mathbf{0}) \neq 0$. Therefore, the quadrupolar coupling can neither preempt nor smear out the bulk nematic phase transition. A nonzero $N(\boldsymbol{q}=\mathbf{0})$ can, however, be induced when the spatial profile of the disorder potential explicitly breaks $C_{4}$ symmetry. This can be seen by including higher-order couplings in the GL free energy (see also the SM [53]):

$$
\delta \mathcal{F}(\boldsymbol{r})=-\left\{g^{\prime} V(\boldsymbol{r})+g^{\prime \prime}[V(\boldsymbol{r})]^{2}\right\}[N(\boldsymbol{r})]^{2} / 2 .
$$

The above terms provide couplings between $V(\boldsymbol{q} \neq \mathbf{0})$ and $N(\boldsymbol{q}=\mathbf{0})$, as well as the $N(\boldsymbol{q} \neq \mathbf{0})$ nematic-field components. 
These couplings are essential to describe a disorder-driven preemptive nematic transition above $T_{\text {nem }}$, as well as the emergence of net nematicity when the potential breaks $C_{4}$ symmetry. To demonstrate both aspects, we derive the modified EOM for the $N(\boldsymbol{q}=\mathbf{0})$ component after adding the contribution of Eq. (5) to the free energy of Eq. (1). We find the following EOM:

$$
\begin{aligned}
\alpha(T & \left.-T_{\mathrm{nem}}\right) N(\boldsymbol{q}=\mathbf{0}) \\
= & g^{\prime} \int d \boldsymbol{p} V(\boldsymbol{p}) N(-\boldsymbol{p}) \\
& +g^{\prime \prime} \iint d \boldsymbol{p} d \boldsymbol{p}^{\prime} V\left(\boldsymbol{p}^{\prime}\right) V\left(\boldsymbol{p}-\boldsymbol{p}^{\prime}\right) N(-\boldsymbol{p}) .
\end{aligned}
$$

Thus, a nonzero $N(\boldsymbol{q}=\mathbf{0})$ can only emerge when components with $\boldsymbol{q} \neq \mathbf{0}$ are already nonzero. By assuming that the potential is weak, the $N(\boldsymbol{q} \neq \mathbf{0})$ components remain given by the Fourier transform of Eq. (2). Therefore, we obtain the following equation up to second order in $V(\boldsymbol{r})$ :

$$
\begin{aligned}
& {\left[\alpha\left(T-T_{\text {nem }}\right)-g^{\prime \prime} \int d \boldsymbol{p}|V(\boldsymbol{p})|^{2}\right] N(\boldsymbol{q}=\mathbf{0})} \\
& \quad=\frac{g g^{\prime}}{c} \int d \boldsymbol{p} \frac{\left(p_{x}^{2}-p_{y}^{2}\right)|V(\boldsymbol{p})|^{2}}{\boldsymbol{p}^{2}+\xi_{\mathrm{nem}}^{-2}}
\end{aligned}
$$

Equation (7) implies that a $C_{4}$-symmetric configuration of impurities cannot source a homogeneous component for the nematic field, since the rhs is zero. As we prove in the SM [53], this holds even after including all the symmetryallowed higher-order GL terms. In fact, this result is also recovered in the case of a large number of randomly distributed and uncorrelated impurities, in which situation, translational, and rotational symmetries are preserved on average. Thus, a $C_{4}$-symmetric disorder potential solely modifies the nematic Stoner criterion, i.e.,

$$
T_{\text {nem }}^{\mathrm{imp}}=T_{\text {nem }}+\frac{g^{\prime \prime}}{\alpha} \int d \boldsymbol{p}|V(\boldsymbol{p})|^{2} .
$$

Depending on the microscopic details which control the sign of the coupling constant $g^{\prime \prime}$, the nematic transition temperature can be enhanced. Note, however, that such an enhancement tends to zero in the thermodynamic limit, unless a critical density of impurities $n_{\text {imp }}$ is present. This is because the $g^{\prime \prime}$ coefficient is inversely proportional to the system size. Interestingly, a detailed transport study with controlled disorder by electron irradiation found cases where the critical nematic transition temperature increased slightly with disorder [54].

Before proceeding, we point out that the first term of Eq. (5) also allows us to describe the induced net nematicity when the disorder potential breaks $C_{4}$ symmetry. To exemplify this, we consider a dimer impurity potential $V(\boldsymbol{r})=V[\delta(\boldsymbol{r}-\hat{\boldsymbol{x}})+\delta(\boldsymbol{r}+\hat{\boldsymbol{x}})]$, which yields $V(\boldsymbol{p})=$ $V\left(\cos p_{x}+\cos p_{y}\right)+V\left(\cos p_{x}-\cos p_{y}\right)$, for a lattice constant $a=1$. The breaking of $C_{4}$ symmetry is ensured by the combined presence of the $A_{1 g}$ and $B_{1 g}$ IRs. In general, a nonzero $N(\boldsymbol{q}=\mathbf{0})$ arises whenever $|V(\boldsymbol{q})|^{2}$ contains at least one $B_{1 g}$ term.

\section{MICROSCOPIC MODEL AND SELF-CONSISTENT CALCULATIONS}

To support the above GL findings, we employ a microscopic tight-binding model of electrons coupled to disorder. This analysis not only verifies the above GL results, but more importantly, allows us to uncover further microscopic details which control the emergence of nematicity. In the absence of disorder, the electrons are described by the dispersion $\varepsilon_{k}=-2 t\left(\cos k_{x}+\cos k_{y}\right)-\mu$. The spin degree of freedom is neglected throughout this work, since it merely introduces a factor of 2 in all thermodynamic averages. We assume that the electrons feel an attractive effective interaction in the Pomeranchuk nematic channel as in Ref. [55], which, after mean-field decoupling, yields the nematic order parameter (for details see the SM [53]):

$$
N_{R}=-V_{\mathrm{nem}} \sum_{\delta} f_{\delta}\left\langle c_{\boldsymbol{R}+\delta}^{\dagger} c_{R}+c_{R}^{\dagger} c_{R+\delta}\right\rangle,
$$

i.e., the lattice analog of $N(\boldsymbol{r})$. This introduces a local or global $C_{4}$ breaking to the electron-hopping matrix elements. In the above, $c_{\boldsymbol{R}}$ denotes the annihilation operator of an electron at position $\boldsymbol{R}=(n, m)$ of the lattice, with $n, m \in \mathbb{Z}$. In addition, $\hat{\boldsymbol{x}}(\hat{\boldsymbol{y}})$ corresponds to the unit vector in the $x(y)$ direction. The nematic form factor is nonzero for $\boldsymbol{\delta}= \pm \hat{\boldsymbol{x}}, \pm \hat{\boldsymbol{y}}$, and reads $f_{ \pm \hat{x}}=-f_{ \pm \hat{y}}=1 / 4$. Disorder is considered in the form of pointlike identical impurities. The total mean-field Hamiltonian becomes

$$
\begin{aligned}
\widehat{\mathcal{H}}= & \sum_{\boldsymbol{R}, \delta}\left(N_{\boldsymbol{R}} f_{\delta}-t / 2\right)\left(c_{\boldsymbol{R}+\delta}^{\dagger} c_{\boldsymbol{R}}+\text { H.c. }\right) \\
& +\sum_{\boldsymbol{R}}\left(V_{\boldsymbol{R}}-\mu\right) c_{\boldsymbol{R}}^{\dagger} c_{\boldsymbol{R}} .
\end{aligned}
$$

For a single delta-function impurity potential $V_{R}=V \delta_{R, 0}$, we evaluate the nematic order parameter in Eq. (9) selfconsistently for a fixed electron density $\langle n\rangle$ (see the SM [53]). The resulting nematic order is displayed in Fig. 2(a), and possesses the same spatial profile as those shown in Fig. 1. In the case of a dimer impurity potential $V_{\boldsymbol{R}}=V\left(\delta_{\boldsymbol{R}, \hat{x}}+\delta_{\boldsymbol{R},-\hat{x}}\right)$, which explicitly breaks $C_{4}$ symmetry, we obtain the profile shown in Fig. 2(c). Its Fourier transform, see Fig. 2(d), exhibits $N_{q=0} \neq 0$, which originates from the rhs of Eq. (7). We stress that the fact that the induced clover pattern in Fig. 2(a) is directly sourced by the EFG, makes it distinct from other microscopic studies of impurity-induced local order [56-58]. There, the impurity potential induces a spontaneous symmetry breaking locally, by means of a local fulfillment of the Stoner criterion, i.e., analogously to Eq. (8).

We proceed by studying the effects of a single impurity for $T<T_{\text {nem }}$, where the system resides in the bulk phase with a value $N_{\mathrm{B}}$ for the nematic order parameter. In this case, the order parameter assumes the form $N(\boldsymbol{r})=N_{\mathrm{B}}+\delta N(\boldsymbol{r})$, where $\delta N(\boldsymbol{r})$ incorporates the spatial variation of the nematic order parameter near the impurity. For a weak impurity potential, we expand the EOM stemming from Eq. (1) up to linear order in $\delta N(\boldsymbol{r})$ (see the SM [53]). We find that $\delta N(\boldsymbol{r})$ possesses the spatial profile of Eq. (3), with the difference that the coherence length is now given by $\xi_{\text {nem }}^{-1}=\sqrt{2 \alpha\left(T_{\text {nem }}-T\right) / c}$ due to an additional contribution of the quartic term which has to be taken into account for $T<T_{\text {nem. }}$. From a microscopic 

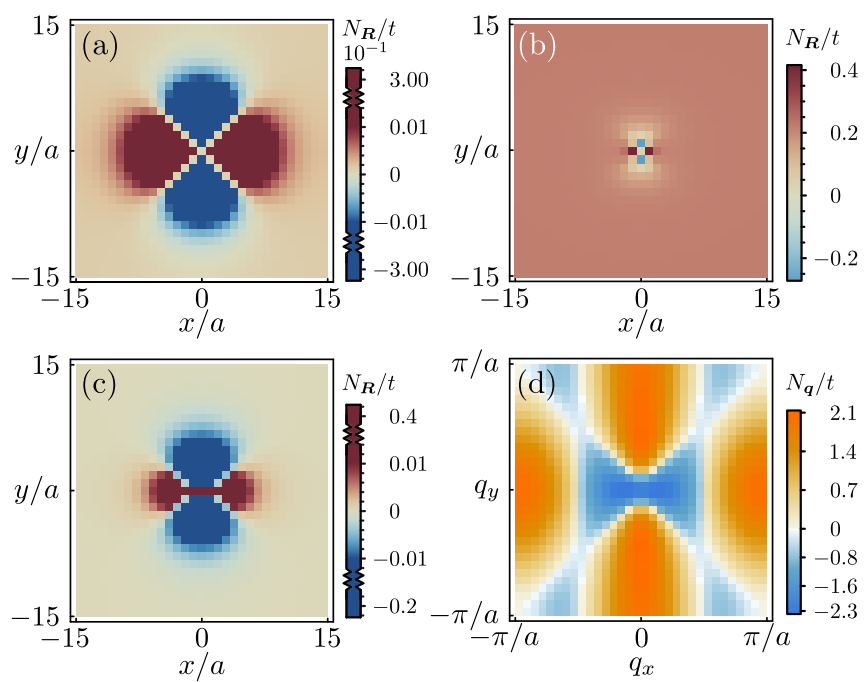

FIG. 2. Numerically obtained nematic order parameter using the microscopic model of Eq. (10): (a) Displays the local nematic order pinned by a single impurity at $\boldsymbol{R}=\mathbf{0}$ for $T=0.8 t$. For the given set of parameters, the Stoner criterion is fulfilled for $T \sim 0.78 t$. (b) Same as in (a), but in the bulk nematic phase $(T=0.76 t)$. (c) Induced nematic order in the presence of a dimer impurity potential, $V_{\boldsymbol{R}}=V\left(\delta_{\boldsymbol{R}, \hat{\boldsymbol{x}}}+\delta_{\boldsymbol{R},-\hat{\boldsymbol{x}}}\right)$, and (d) its discrete Fourier transform $(T=0.8 t)$. From $(\mathrm{d})$, one clearly sees that the breaking of $C_{4}$ symmetry indeed induces $N_{q=0} \neq 0$. All the figures were obtained using: $V=5 t, \mathcal{N}_{x}=\mathcal{N}_{y}=31, V_{\text {nem }}=4 t, k_{\mathrm{B}}=1$, and $\langle n\rangle=0.53$.

calculation, we obtain the spatial profile for the nematic order parameter which is shown in Fig. 2(b), exhibiting an anisotropic local structure which is slightly elongated along the $y$ direction. To lowest order in $V(\boldsymbol{r})$, this asymmetry found via the microscopic model can be reproduced in the GL theory by including the first term of Eq. (5). The presence of this term yields $\delta N(\boldsymbol{r}) \propto f(r) \cos (2 \phi)+h(r) N_{\mathrm{B}}$, where $f(r)$ and $h(r)$ are decaying functions of the radial coordinate, transforming according to the $A_{1 g}$ IR. Note that additional higher order terms, e.g., $\propto V(\boldsymbol{r})\left(\partial_{x}^{2}+\partial_{y}^{2}\right)[N(\boldsymbol{r})]^{2}$, can further contribute to this anisotropy by modifying $h(r)$. In general, we find that depending on the sign of the impurity potential, pointlike disorder at $T<T_{\text {nem }}$ may either locally enhance or decrease the nematic order.

Finally, we verify the possibility of disorder-enhanced $T_{\text {nem }}$ within the microscopic model. We assume random and dilute disorder of density $n_{\text {imp }}$, that on average preserves the $C_{4}$ symmetry. Within the first order Born approximation [59], the quasiparticle lifetime is

$$
\frac{1}{\tau_{k}}=2 \pi n_{\mathrm{imp}} V^{2} \frac{1}{\mathcal{N}} \sum_{p} \delta_{\varepsilon_{p}, \varepsilon_{k}} .
$$

By use of Eq. (11), we can evaluate the microscopic coefficients which enter the modified Stoner criterion of Eq. (8), brought about by the impurities. Starting from Eq. (9), we find that the self-consistency equation for the $\boldsymbol{q}=\mathbf{0}$ component of the nematic mean-field order parameter, corresponding to net nematicity $N \equiv \sum_{\boldsymbol{R}} N_{\boldsymbol{R}} / \mathcal{N}=N_{\boldsymbol{q}=\mathbf{0}} / \mathcal{N}$, reads

$$
N=-V_{\mathrm{nem}} \frac{1}{\mathcal{N}} \sum_{\boldsymbol{k}} f_{\boldsymbol{k}} \int_{-\infty}^{+\infty} \frac{d \varepsilon}{2 \pi} \frac{n_{F}\left(\varepsilon+\varepsilon_{\boldsymbol{k}}+N f_{k}\right)}{\varepsilon^{2}+1 /\left(2 \tau_{\boldsymbol{k}}\right)^{2}} \frac{1}{\tau_{\boldsymbol{k}}},
$$

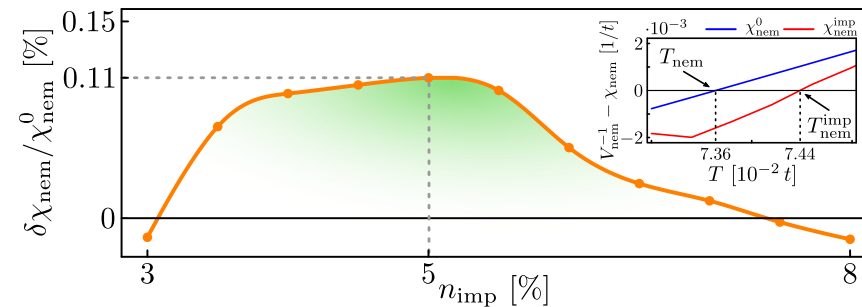

FIG. 3. Relative disorder-induced modification of the nematic susceptibility $\delta \chi_{\text {nem }} / \chi_{\text {nem }}^{0}=\left(\chi_{\text {nem }}^{\text {imp }}-\chi_{\text {nem }}^{0}\right) / \chi_{\text {nem }}^{0}$ versus the disorder concentration $n_{\text {imp }}$. Here $\chi_{\text {nem }}^{0}$ is the rhs of Eq. (12) in the absence of disorder $\tau_{k} \rightarrow \infty$. The inset shows that the $T_{\text {nem }}$ increases by approximately $1 \%$ for $n_{\mathrm{imp}} \approx 5 \%$, due to the disorder-modified Stoner criterion. Parameters: $V_{\text {nem }}=1.584 t,\langle n\rangle=0.53, \mathcal{N}_{x}=\mathcal{N}_{y}=201$, $T=0.075 t$, and $V=5 t$.

with $f_{k}=\cos k_{x}-\cos k_{y}$ and $n_{F}(\varepsilon)$ the Fermi-Dirac distribution function. Linearizing the rhs with respect to $N$, yields the modified Stoner criterion

$$
\frac{1}{V_{\mathrm{nem}}}=-\frac{1}{\mathcal{N}} \sum_{k} f_{k}^{2} \int_{-\infty}^{+\infty} \frac{d \varepsilon}{2 \pi} \frac{n_{F}^{\prime}\left(\varepsilon+\varepsilon_{k}\right)}{\varepsilon^{2}+1 /\left(2 \tau_{k}\right)^{2}} \frac{1}{\tau_{k}} .
$$

In the absence of disorder, i.e., $\tau_{k} \rightarrow \infty$, the integration yields the derivative of the Fermi-Dirac distribution $n_{F}^{\prime}\left(\varepsilon_{k}\right)$. However, for finite $\tau_{k}$, each $\boldsymbol{k}$ state is broadened, and the density of states (DOS) for the $\boldsymbol{k}$ points mainly contributing to the nematic instability may be enhanced. To explore this effect, we numerically calculate the nematic susceptibility $\chi_{\text {nem }}^{\text {imp }}$ in the presence of disorder, which is identified with the rhs of Eq. (12). Figure 3 shows how this quantity changes versus the disorder concentration $n_{\text {imp }}$, relative to the disorder-free case. For an impurity density of $n_{\text {imp }} \approx 5 \%$, we obtain the maximal relative enhancement of $\chi_{\text {nem }}^{\text {imp leading to }}$ a corresponding small enhancement of $T_{\text {nem }}$. It is tempting to assign the similar small enhancement of the nematic transition temperature measured experimentally in Ref. [54] to the effect demonstrated in Fig. 3.

The origin of the enhancement effect shown in Fig. 3 is the presence of a nearby van Hove singularity whose spectral weight can be utilized to boost $\chi_{\text {nem }}^{\text {imp }}$ in the presence of finite $\tau_{k}$. Without favorable DOS conditions, disorder generally suppresses the nematic susceptibility and hence also $T_{\text {nem }}$. Such a suppression tendency has been previously found in Ref. [60] and is also demonstrated in our SM [53]. Furthermore, we remark that even in the disorder-free case, the presence of a van Hove singularity is pivotal for the stabilization of an electron nematic phase of the Pomeranchuk type. For more details see Refs. [61,62].

\section{SUMMARY}

In summary, we have elucidated the coupling of nematicity to disorder from both a phenomenological GL approach and microscopic calculations. Importantly, disorder is always locally relevant for inducing nematicity since the EFG $\left(\partial_{x}^{2}-\partial_{y}^{2}\right) V(\boldsymbol{r})$ directly acts as a quadrupolar source 
field for nematicity. This explains the detection of local nematicity/orthorhombicity in experimental probes sensitive to different atomic environments within materials. At the global scale, however, disorder does not generally generate long-range nematicity at $T>T_{\text {nem }}$ where the system remains tetragonal. Finite disorder concentrations may, however, under favorable circumstances enhance nematic order.

\section{ACKNOWLEDGMENTS}

We thank A. Kreisel and K. Flensberg for useful discussions. D.S. and B.M.A. acknowledge financial support from the Carlsberg Foundation. P.K. and B.M.A. acknowledge support from the Independent Research Fund Denmark Grant No. DFF-6108-00096. I.P. is supported by ANR Grant "IRONIC" (ANR-15-CE30-0025).
[1] E. Fradkin, S. A. Kivelson, M. J. Lawler, J. P. Eisenstein, and A. P. Mackenzie, Annu. Rev. Condens. Matter Phys. 1, 153 (2010).

[2] M. P. Lilly, K. B. Cooper, J. P. Eisenstein, L. N. Pfeiffer, and K. W. West, Phys. Rev. Lett. 82, 394 (1999).

[3] R. R. Du, D. C. Tsui, H. L. Stormer, L. N. Pfeiffer, K. W. Baldwin, and K. W. West, Solid State Commun. 109, 389 (1999).

[4] R. A. Borzi, S. A. Grigera, J. Farrell, R. S. Perry, S. J. S. Lister, S. L. Lee, D. A. Tennant, Y. Maeno, and A. P. Mackenzie, Science 315, 214 (2017).

[5] V. Hinkov, D. Haug, B. Fauqué, P. Bourges, Y. Sidis, A. Ivanov, C. Bernhard, C. T. Lin, and B. Keimer, Science 319, 597 (2008).

[6] S. Yonezawa, K. Tajiri, S. Nakata, Y. Nagai, Z. Wang, K. Segawa, Y. Ando, and Y. Maeno, Nat. Phys. 13, 123 (2017).

[7] R. Tao, Y.-J. Yan, X. Liu, Z.-W. Wang, Y. Ando, Q.-H. Wang, T. Zhang, and D.-L. Feng, Phys. Rev. X 8, 041024 (2018).

[8] S. Yonezawa, Condens. Matter 4, 2 (2019).

[9] M. A. Tanatar, E. C. Blomberg, A. Kreyssig, M. G. Kim, N. Ni, A. Thaler, S. L. Budko, P. C. Canfield, A. I. Goldman, I. I. Mazin, and R. Prozorov, Phys. Rev. B 81, 184508 (2010).

[10] J.-H. Chu, J. G. Analytis, K. De Greve, P. L. McMahon, Z. Islam, Y. Yamamoto, and I. R. Fisher, Science 329, 824 (2010).

[11] J. J. Ying, X. F. Wang, T. Wu, Z. J. Xiang, R. H. Liu, Y. J. Yan, A. F. Wang, M. Zhang, G. J. Ye, P. Cheng, J. P. Hu, and X. H. Chen, Phys. Rev. Lett. 107, 067001 (2011).

[12] J.-H. Chu, H.-H. Kuo, J. G. Analytis, and I. R. Fisher, Science 337, 710 (2012).

[13] E. C. Blomberg, M. A. Tanatar, R. M. Fernandes, I. I. Mazin, B. Shen, H.-H. Wen, M. D. Johannes, J. Schmalian, and R. Prozorov, Nat. Commun. 4, 1914 (2013).

[14] S. Ishida, M. Nakajima, T. Liang, K. Kihou, C. H. Lee, A. Iyo, H. Eisaki, T. Kakeshita, Y. Tomioka, T. Ito, and S. Uchida, Phys. Rev. Lett. 110, 207001 (2013); J. Am. Chem. Soc. 135, 3158 (2013)

[15] H.-H. Kuo and I. R. Fisher, Phys. Rev. Lett. 112, 227001 (2014).

[16] M. Yi, D. Lu, J.-H. Chu, J. G. Analytis, A. P. Sorini, A. F. Kemper, B. Moritz, S.-K. Mo, R. G. Moore, M. Hashimoto, W.-S. Lee, Z. Hussain, T. P. Devereaux, I. R. Fisher, and Z.-X. Shen, Proc. Natl. Acad. Sci. USA 108, 6878 (2011).

[17] A. Kostin, P. O. Sprau, A. Kreisel, Y. X. Chong, A. E. Böhmer, P. C. Canfield, P. J. Hirschfeld, B. M. Andersen, and J. C. Davis, Nat. Mater. 17, 869 (2018).

[18] J. Zhao, D. T. Adroja, D.-X. Yao, R. Bewley, S. Li, X. F. Wang, G. Wu, X. H. Chen, J. Hu, and P. Dai, Nat. Phys. 5, 555 (2009)
[19] M. Nakajima, T. Liang, S. Ishida, Y. Tomioka, K. Kihou, C. H. Lee, A. Iyo, H. Eisaki, T. Kakeshita, T. Ito, and S. Uchida, Proc. Natl. Acad. Sci. USA 108, 12238 (2011).

[20] A. Dusza, A. Lucarelli, F. Pfuner, J. H. Chu, I. R. Fisher, and L. Degiorgi, Europhys. Lett. 93, 37002 (2011).

[21] H. Z. Arham, C. R. Hunt, W. K. Park, J. Gillett, S. D. Das, S. E. Sebastian, Z. J. Xu, J. S. Wen, Z. W. Lin, Q. Li, G. Gu, A. Thaler, S. Ran, S. L. Bud'ko, P. C. Canfield, D. Y. Chung, M. G. Kanatzidis, and L. H. Greene, Phys. Rev. B 85, 214515 (2012).

[22] S. Kasahara, H. J. Shi, K. Hashimoto, S. Tonegawa, Y. Mitzukami, T. Shibauchi, K. Sugimoto, T. Fukuda, T. Terashima, A. H. Nevidomskyy, and Y. Matsuda, Nature (London) 486, 382 (2012).

[23] A. Kerelsky, L. McGilly, D. M. Kennes, L. Xian, M. Yankowitz, S. Chen, K. Watanabe, T. Taniguchi, J. Hone, C. Dean, A. Rubio, and A. N. Pasupathy, Nature (London) 572, 95 (2019).

[24] Y. Gallais, R. M. Fernandes, I. Paul, L. Chauvière, Y.-X. Yang, M.-A. Méasson, M. Cazayous, A. Sacuto, D. Colson, and A. Forget, Phys. Rev. Lett. 111, 267001 (2013).

[25] R. M. Fernandes, A. V. Chubukov, and J. Schmalian, Nat. Phys. 10, 97 (2014).

[26] A. E. Böhmer and A. Kreisel, J. Phys. Condens. Matter 30, 023001 (2018).

[27] T.-M. Chuang, M. P. Allan, J. Lee, Y. Xie, N. Ni, S. L. Bud'ko, G. S. Boebinger, P. C. Canfield, and J. C. Davis, Science 327, 181 (2010).

[28] C.-L. Song, Y.-L. Wang, P. Cheng, Y.-P. Jiang, W. Li, T. Zhang, Z. Li, K. He, L. Wang, J.-F. Jia, H.-H. Hung, C. Wu, X. Ma, X. Chen, and Q.-K. Xue, Science 332, 1410 (2011).

[29] X. Zhou, C. Ye, P. Cai, X. Wang, X. Chen, and Y. Wang, Phys. Rev. Lett. 106, 087001 (2011).

[30] S. Grothe, S. Chi, P. Dosanjh, R. Liang, W. N. Hardy, S. A. Burke, D. A. Bonn, and Y. Pennec, Phys. Rev. B 86, 174503 (2012).

[31] M. P. Allan, T.-M. Chuang, F. Massee, Y. Xie, N. Ni, S. L. Bud'ko, G. S. Boebinger, Q. Wang, D. S. Dessau, P. C. Canfield, M. S. Golden, and J. C. Davis, Nat. Phys. 9, 220 (2013).

[32] E. P. Rosenthal, E. F. Andrade, C. J. Arguello, R. M. Fernandes, L. Y. Xing, X. C. Wang, C. Q. Jin, A. J. Millis, and A. N. Pasupathy, Nat. Phys. 10, 225 (2014).

[33] X. Ren, L. Duan, Y. Hu, J. Li, R. Zhang, H. Luo, P. Dai, and Y. Li, Phys. Rev. Lett. 115, 197002 (2015).

[34] S.-H. Baek, D. V. Efremov, J. M. Ok, J. S. Kim, J. van den Brink, and B. Büchner, Phys. Rev. B 93, 180502(R) (2016).

[35] M. N. Gastiasoro, I. Paul, Y. Wang, P. J. Hirschfeld, and B. M. Andersen, Phys. Rev. Lett. 113, 127001 (2014). 
[36] Y. Wang, M. N. Gastiasoro, B. M. Andersen, M. Tomić, H. O. Jeschke, R. Valentí, I. Paul, and P. J. Hirschfeld, Phys. Rev. Lett. 114, 097003 (2015).

[37] C.-C. Chen, B. Moritz, J. van den Brink, T. P. Devereaux, and R. R. P. Singh, Phys. Rev. B 80, 180418(R) (2009).

[38] Y. Inoue, Y. Yamakawa, and H. Kontani, Phys. Rev. B 85, 224506 (2012).

[39] M. N. Gastiasoro, P. J. Hirschfeld, and B. M. Andersen, Phys. Rev. B 89, 100502(R) (2014).

[40] M. Hoyer, R. M. Fernandes, A. Levchenko, and J. Schmalian, Phys. Rev. B 93, 144414 (2016).

[41] T. Iye, M.-H. Julien, H. Mayaffre, M. Horvatić, C. Berthier, K. Ishida, H. Ikeda, S. Kasahara, T. Shibauchi, and Y. Matsuda, J. Phys. Soc. Jpn. 84, 043705 (2015).

[42] R. Zhou, L. Y. Xing, X. C. Wang, C. Q. Jin, and G.-Q. Zheng, Phys. Rev. B 93, 060502(R) (2016).

[43] P. S. Wang, P. Zhou, S. S. Sun, Y. Cui, T. R. Li, H. Lei, Z. Wang, and W. Yu, Phys. Rev. B 96, 094528 (2017).

[44] P. Wiecki, M. Nandi, A. E. Böhmer, S. L. Bud'ko, P. C. Canfield, and Y. Furukawa, Phys. Rev. B 96, 180502(R) (2017).

[45] M. Toyoda, Y. Kobayashi, and M. Itoh, Phys. Rev. B 97, 094515 (2018).

[46] W. Wang et al., Nat. Commun. 9, 3128 (2018).

[47] P. Zhang, T. Qian, P. Richard, X. P. Wang, H. Miao, B. Q. Lv, B. B. Fu, T. Wolf, C. Meingast, X. X. Wu, Z. Q. Wang, J. P. Hu, and H. Ding, Phys. Rev. B 91, 214503 (2015).

[48] C.-W. Luo, P. C. Cheng, S.-H. Wang, J.-C. Chiang, J.-Y. Lin, K.-H. Wu, J.-Y. Juang, D. A. Chareev, O. S. Volkova, and A. N. Vasiliev, npj Quantum Mater. 2, 32 (2017).

[49] R. J. Koch, T. Konstantinova, M. Abeykoon, A. Wang, C. Petrovic, Y. Zhu, E. S. Bozin, and S. J. L. Billinge, Phys. Rev. B 100, 020501(R) (2019).
[50] B. A. Frandsen, Q. Wang, S. Wu, J. Zhao, and R. J. Birgeneau, Phys. Rev. B 100, 020504(R) (2019).

[51] G. Livanas, A. Aperis, P. Kotetes, and G. Varelogiannis, Phys. Rev. B 91, 104502 (2015).

[52] R. M. Fernandes and A. J. Millis, Phys. Rev. Lett. 111, 127001 (2013).

[53] See Supplemental Material at http://link.aps.org/supplemental/ 10.1103/PhysRevB.100.064521 for details on the GinzburgLandau theory and the microscopic model, including derivation of the mean-field Hamiltonian and further results for the disorder-modified Stoner criterion.

[54] E. I. Timmons, M. A. Tanatar, K. Willa, S. Teknowijoyo, K. Cho, M. Kończykowski, O. Cavani, Y. Liu, T. A. Lograsso, U. Welp, and R. Prozorov, Phys. Rev. B 99, 054518 (2019).

[55] Y. Gallais and I. Paul, C. R. Phys. 17, 113 (2016).

[56] B. M. Andersen, P. J. Hirschfeld, A. P. Kampf, and M. Schmid, Phys. Rev. Lett. 99, 147002 (2007).

[57] A. T. Rømer, S. Graser, T. S. Nunner, P. J. Hirschfeld, and B. M. Andersen, Phys. Rev. B 86, 054507 (2012).

[58] M. N. Gastiasoro, P. J. Hirschfeld, and B. M. Andersen, Phys. Rev. B 88, 220509(R) (2013).

[59] H. Bruus and K. Flensberg, Many-Body Quantum Theory in Condensed Matter Physics (Oxford University Press, Oxford, 2004).

[60] A. F. Ho and A. J. Schofield, Europhys. Lett. 84, 27007 (2008).

[61] I. Khavkine, C.-H. Chung, V. Oganesyan, and H.-Y. Kee, Phys. Rev. B 70, 155110 (2004).

[62] H. Yamase, V. Oganesyan, and W. Metzner, Phys. Rev. B 72, 035114 (2005). 